\title{
The Greek Alphabet
}

The Greek alphabet is used throughout this volume, because the actual pronunciation varies enormously, both diachronically and diatopically, which would have resulted in different transcriptions for same or similar words from different periods. For the benefit of readers who are unfamiliar with the Greek alphabet or unfamiliar with either the ancient $\left(5^{\text {th }}-4^{\text {th }} \mathrm{c}\right.$. BC) or the modern pronunciation we provide here a comparative table of both. The modern pronunciation is basically the same as that of the LMedGr, barring diatopic variation. The successive changes in the pronunciation of the Greek vowels and diphthongs are the most complex. For detailed information on these changes with further bibliographical references we refer the interested reader to Horrocks (2010). ${ }^{1}$ The following tables are based on Horrocks (2010: xviii-xx).

\section{Greek letter}

A $\alpha$

B $\beta$

Г $\gamma$

$\Delta \quad \delta$

E $\varepsilon$

Z $\zeta$

$\mathrm{H} \quad \eta$

$\Theta \quad \theta$

I 1

K $\kappa$

$\Lambda \quad \lambda$

M $\mu$

$\mathrm{N} \quad v$

$\Xi \quad \xi$

O $\mathrm{O}$

$\Pi \pi$

P $\rho$

$\Sigma \quad \sigma, \varsigma$

$\mathrm{T} \tau$

Y v

$\Phi \quad \varphi$ alpha

beta

gamma

delta

epsilon

zeta

eta

theta

iota

kappa

lambda

$\mathrm{mu}$

nu

xi

omikron

pi

rho

sigma

tau

upsilon

phi

\section{Ancient pronunciation}

[a], [a:]

[b]

[g]

[d]

[e]

[dz], [zd]

[ع:]

[th]

[i], [i:]

[k]

[1]

[m]

[n]

[ks]

[o]

[p]

[r]

[s]

[t]

[y], [y:]

$\left[\mathrm{p}^{\mathrm{h}}\right]$
Modern pronunciation

[a]

[v]

[४], [j]

[ð]

[e]

[z]

[i]

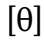

[i]

[k], [c]

[1]

[m]

[n]

[ks]

[o]

[p]

[r]

[s]

[t]

[i]

[f]

1 Horrocks, Geoffrey C. 2010. Greek: A history of the language and its speakers, $2^{\text {nd }}$ ed. Malden: Wiley-Blackwell. 


$\begin{array}{lllll}\mathrm{X} & \chi & \text { khi } & {\left[\mathrm{k}^{\mathrm{h}}\right]} & {[\mathrm{x}],[\mathrm{c}]} \\ \Psi & \psi & \text { psi } & {[\mathrm{ps}]} & {[\mathrm{ps}]} \\ \Omega & \omega & \text { omega } & {[\mathrm{o}:]} & {[\mathrm{o}]}\end{array}$

\begin{tabular}{|c|c|c|}
\hline $\begin{array}{l}\text { Digraphs } \\
\alpha \mathrm{l}\end{array}$ & $\begin{array}{l}\text { Ancient pronunciation } \\
{[\mathrm{aI}]}\end{array}$ & $\begin{array}{l}\text { Modern pronunciation } \\
{[\mathrm{e}]}\end{array}$ \\
\hline$\alpha u$ & [av] & [af], [av] \\
\hline$\varepsilon l$ & [e:] & [i] \\
\hline$\varepsilon v$ & [eu] & [ef], [ev] \\
\hline ot & [oI] & [i] \\
\hline ov & [u:] & {$[u]$} \\
\hline$\alpha$ & [a:I] & [a] \\
\hline$n$ & {$[\varepsilon: I]$} & [i] \\
\hline$\omega$ & [o:I] & [o] \\
\hline yy & [ng] & {$[(\mathrm{n}) \mathrm{g}]$} \\
\hline$y \kappa$ & [nk] & {$[(\mathrm{n}) \mathrm{g}]$} \\
\hline$y x$ & {$\left[\mathrm{nk}^{\mathrm{h}}\right]$} & [nx], [nç] \\
\hline$\mu \pi$ & [mp] & {$[(\mathrm{m}) \mathrm{b}]$} \\
\hline$v \tau$ & [nt] & {$[(\mathrm{n}) \mathrm{d}]$} \\
\hline
\end{tabular}

\section{Diacritics}

, smooth breathing

- rough breathing

- acute accent

- grave accent

- circumflex

\section{Ancient pronunciation}

$\emptyset$

[h]

[rise + fall on next syllable] [absence of rise]

[rise-fall]
Modern pronunciation

$\emptyset$

$\emptyset$

[stress]

[stress]

[stress] 